\title{
Medullary thyroid carcinoma (MTC): unusual metastatic sites
}

\author{
Paraskevi Kazakou, George Simeakis, Maria Alevizaki and Katerina Saltiki \\ Endocrine Unit, Department of Clinical Therapeutics, Alexandra Hospital, School of Medicine, National and \\ Kapodistrian University of Athens, Athens, Greece
}

Correspondence should be addressed to P Kazakou

Email

vkazakou@hotmail.com

\section{Summary}

Medullary thyroid carcinoma (MTC) has a varying clinical course; distant metastases are frequently present even at diagnosis. We present two MTC cases with unusual metastatic sites. Two female patients are presented with slow progressive MTC. The first case developed distant metastases 23 years after diagnosis and underwent locoregional therapies. At the same time a breast mass developed representing MTC metastasis. Treatment with vandetanib led to long-term disease stabilization. The second patient is presented with metastases in the pancreas 13 years after diagnosis. Shortly, a painful mass developed in the mandible and metastasis of MTC was diagnosed. Disease progression was recorded 20 months after the initiation of local and systemic therapy. Such cases have only rarely been reported in the literature and highlight the need for prompt recognition of unexpected MTC metastases.

\section{Learning points:}

- Unusual sites of metastasis may appear in patients with medullary thyroid carcinoma (MTC) years after the initial diagnosis.

- Although rare, unexpected MTC metastases highlight the need for prompt recognition and appropriate treatment.

- Local recurrences accompanied by inappropriately low calcitonin levels should prompt further investigation for possible distant metastatic disease.

- Systemic treatment with tyrosine kinase inhibitors may be effective even in patients with unusual metastases from MTC.

\section{Background}

Medullary thyroid carcinoma (MTC) is a rare thyroid tumor (3-5\% of thyroid carcinomas) which derives from the thyroid parafollicular C-cells that secrete calcitonin. Ten-year survival rates range from 61 to $88 \%$ depending on the stage at diagnosis. MTC occurs as sporadic form (in $75 \%$ of the cases) and in familial form (in $25 \%$ of the cases) in the context of multiple endocrine neoplasia type-2 syndromes (MEN2); germ-line mutations in the 'rearranged during transfection' (RET) proto-oncogene are responsible for the inherited cases. The clinical course of MTC varies. Regional lymph node metastases are frequent and in $10 \%$ of the cases distant metastases may be present already at diagnosis; liver, lungs, and bone are the most common metastatic sites. Unusual sites of metastases have also been reported (1). Herein, we describe two MTC cases who are presented with very rare metastases in the breast, pancreas, and mandible.

\section{Case presentation 1}

A 71-year-old female with a 23-year history of sporadic MTC and progressive metastatic disease was referred 
to our department for further management. MTC had been diagnosed in 1990 after total thyroidectomy for multinodular goiter. Histological examination revealed a medullary thyroid cancer, $2.5 \mathrm{~cm}$ in diameter, with capsular invasion (T2N×M0). Genetic testing for all the known RET mutations was negative. One year later, bilateral cervical lymph node dissection was performed. Eleven years later, further disease progression in the cervical and mediastinal lymph nodes was recorded and the patient underwent modified neck and mediastinum lymph node dissection. Biochemical and structural disease progressed slowly during the following 13 years; calcitonin levels were elevated and increased slowly over time. In 2013, further progression of calcitonin levels was recorded and new metastases appeared; the calcitonin doubling time was 12 months.

\section{Investigation and treatment}

At this time, she was referred to our department. Serum calcitonin levels were 18,348 pg/mL (normal $<10 \mathrm{pg} /$ $\mathrm{mL}$ ) and carcinoembryonic-antigen (CEA) $176 \mathrm{ng} / \mathrm{mL}$ (normal $<5.1 \mathrm{ng} / \mathrm{mL}$ ). MRI revealed multiple metastases in the cervical lymph-nodes, mediastinum ( $3.6 \mathrm{~cm}$ block), multiple lesions in the liver (maximum dimension: 2.6 $\mathrm{cm}$ ), as well as bone lesions at the thoracic region of the spine. The patient underwent external bone radiotherapy (30 Gy) and liver chemoembolization for reduction of the disease burden. Indeed, 6 months later, serum calcitonin and CEA levels remained slightly elevated. Imaging revealed shrinkage of the liver metastases now measuring up to $1.7 \mathrm{~cm}$ as well as stabilization of the mediastinal mass and bone lesions. However, during the mediastinum MRI, a well marginated oval lesion of $1.3 \mathrm{~cm}$ in the right breast was noted. Further investigation with mammography showed a $1 \mathrm{~cm}$ nodule with regular margins in the upper inner quadrant of the right breast (Fig. 1). A wide local excision of the right breast lump was performed. Paraffin sections and immunohistochemistry revealed that the breast lump corresponded to metastasis of the known MTC; there was no axillary lymph node involvement. Immunohistochemical staining of the resected specimen was positive for calcitonin, CEA, synaptophysin (SYN), chromogranin A ( $\mathrm{CgA})$, and thyroid transcription factor (TTF)-1, but negative for estrogen receptor (ER), progesterone receptor (PR), and gross cystic disease fluid protein (GCDFP)-15. The breast lesion had a diameter of 1 $\mathrm{cm}$, the Ki index was 7\%, the differentiation was Grade 2, and there was no external tissue infiltration.

During a 6-month follow up, calcitonin level increased up to $22472 \mathrm{pg} / \mathrm{mL}$, CEA to $185 \mathrm{ng} / \mathrm{mL}$, and liver metastases

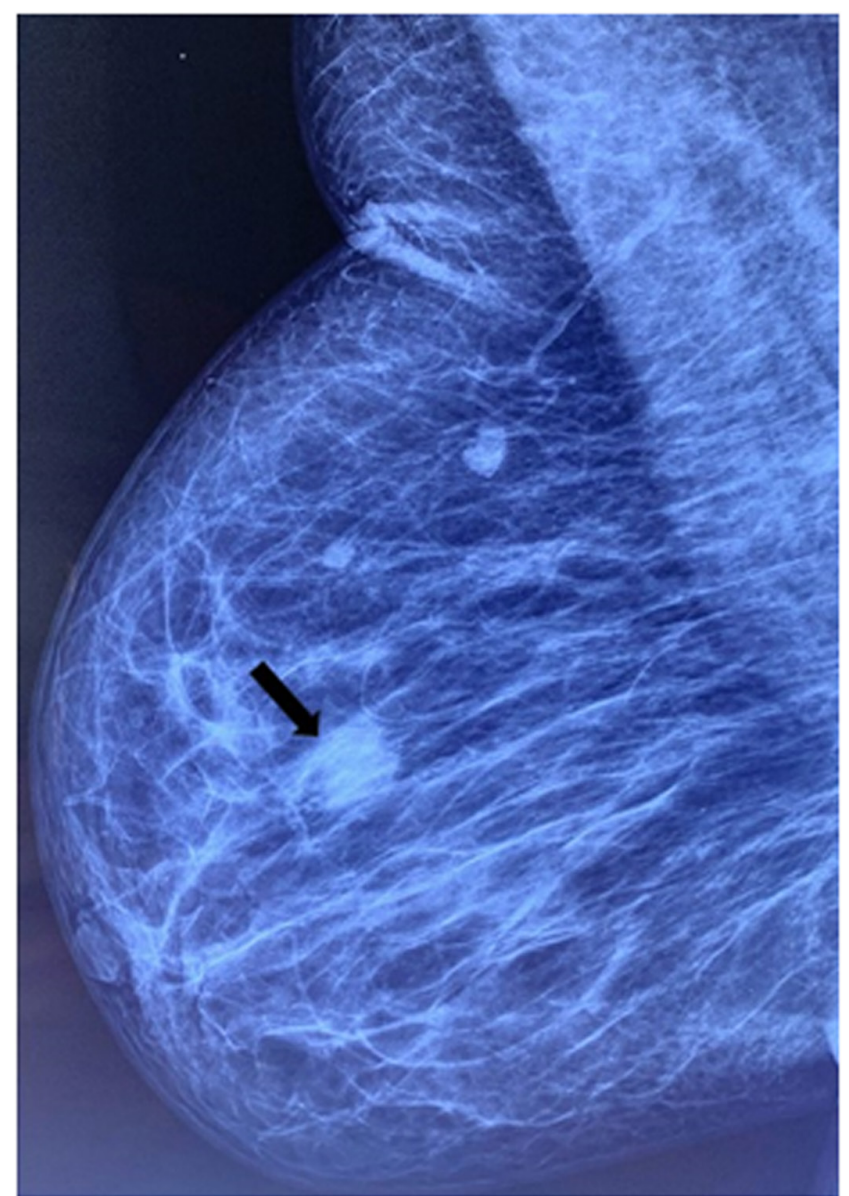

Figure 1

Mammography showing an oval to round, high density lesion with no microcalcifications (MTCmetastasis) (black arrow).

showed further enlargement. At that point, treatment with the tyrosine kinase inhibitor (TKI) vandetanib was initiated, at a maximum dose of $300 \mathrm{mg}$ per day.

\section{Outcome and follow-up}

After a follow-up of further 7 years, the patient is alive; she receives treatment with vandetanib at $300 \mathrm{mg}$ per day which is well tolerated. Calcitonin and CEA levels have decreased to $9790 \mathrm{pg} / \mathrm{mL}$ and $70 \mathrm{ng} / \mathrm{mL}$, respectively; the disease remains stable according to Response Evaluation Criteria in Solid Tumors (RECIST) under vandetanib treatment.

\section{Case presentation 2}

A 61-year-old female with a 6-year history of sporadic MTC was presented to our department for further evaluation. The patient had undergone total thyroidectomy with 
cervical lymph node dissection in 2003. Preoperative basal serum calcitonin was $120 \mathrm{pg} / \mathrm{mL}$. Pathological examination revealed a medullary thyroid carcinoma of $3.7 \mathrm{~cm}$ with capsular invasion; eight out of 19 removed lymph nodes were infiltrated (stage IVA, T4aN1bM0). The 3-month postoperative calcitonin was within normal levels. During the following 6-years she underwent three consecutive bilateral neck lymph node dissections due to locoregional recurrence. It is interesting that despite the metastases the calcitonin levels remained low $(20-40 \mathrm{pg} / \mathrm{mL})$.

\section{Investigation and treatment}

At presentation and during the initial evaluation in our department in January 2009, the patient had slightly increased serum calcitonin levels $(31 \mathrm{pg} / \mathrm{mL}$ ), while CEA levels were normal; imaging was negative for metastatic disease (including neck ultrasound, chest CT, mediastinum, and abdomen MRI and bone scan). Genetic testing for all the known RET mutations was negative. Six years later she presented structural disease progression; new metastases in the left cervical lymph nodes were visualised and the patient underwent elective left lymph node neck dissection. Few months later the patient complained of increasing pain in the right shoulder. A bone scan was performed and showed a new bone metastatic lesion on the right shoulder. The shoulder MRI confirmed a lytic lesion of $2.5 \mathrm{~cm}$. Serum calcitonin and CEA levels at that time were inappropriately low, 138 $\mathrm{pg} / \mathrm{mL}$ and $7 \mathrm{ng} / \mathrm{mL}$, respectively. Fine-needle aspiration biopsy (FNAb) of the lesion revealed metastatic disease from the known MTC and the patient underwent external radiation therapy (30 Gy).

Six-months later, re-staging revealed further progression in the left supraclavicular lymph nodes (maximum dimension of $2.3 \mathrm{~cm}$ ) while the abdominal MRI showed a $6 \mathrm{~cm}$, rounded, and well-delineated lesion in the pancreatic head(Fig. 2). Endoscopic ultrasound (EUS) revealed two further lesions in the pancreatic tail (measuring 2 and $1.3 \mathrm{~cm}$, respectively). EUS guided FNA biopsy confirmed MTC metastasis in all with positive immunohistochemical staining for chromogranin-A, synaptophysin, and calcitonin. Despite the increased tumor burden, serum calcitonin and CEA levels were not markedly elevated ( $426 \mathrm{pg} / \mathrm{mL}$ and $24 \mathrm{ng} / \mathrm{mL}$, respectively) indicating a low secretory tumor. Treatment with vandetanib, $300 \mathrm{mg}$ daily, was initiated.

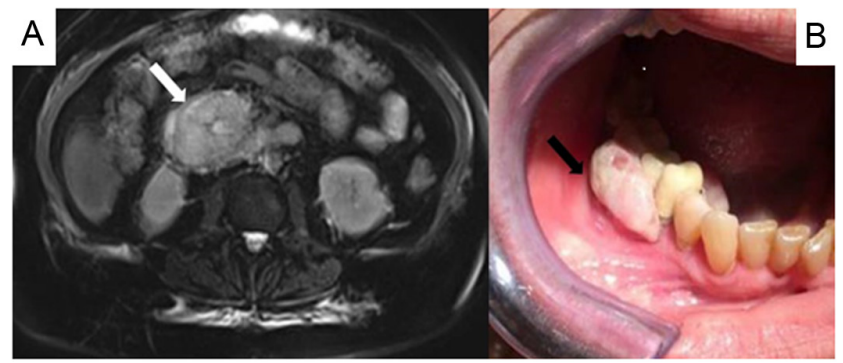

\section{Figure 2}

(A) Abdominal MRI showing the MTC metastatic lesion in the pancreas (white arrow). (B) MTC metastatic lesion in the right mandible (black arrow).

\section{Outcome and follow-up}

During the following 12 months, laboratory examination and repeat imaging was compatible with disease stabilization. However, in September 2016, while under vandetanib treatment, the patient complained of swelling and pain in the right mandible (Fig. 2). CT of the mandible revealed a cystic lytic lesion which was subsequently resected. Histology revealed MTC metastasis. Although no further disease progression was recorded for a total of 20 months under vandetanib treatment, serious deterioration occurred due to upper gastrointestinal hemorrhage. She finally succumbed to the disease after a few months.

\section{Discussion}

MTC is an uncommon and aggressive form of thyroid cancer. Therefore, early identification, appropriate surgical intervention and careful postoperative surveillance are crucial. During the last 2 decades, the application of routine serum calcitonin measurement in thyroid nodular disease, the use of high-resolution ultrasound as well as the genetic screening in familial MTC cases have changed the disease prognosis allowing the diagnosis and treatment at an earlier stage. Total thyroidectomy with cervical lymph node dissection is recommended, depending on preoperative serum calcitonin levels and US findings. According to current guidelines, MTC patients with no evidence of neck lymph node metastases in the ultrasound should undergo total thyroidectomy and central lymph node dissection; in cases with positive imaging, dissection of the central lymph node compartment and the involved lateral neck compartments is recommended. Contralateral neck dissection should be considered if the basal preoperative serum calcitonin levels are $>200 \mathrm{pg} / \mathrm{mL}$ (1). In our country the change in managing policies around the year 2000 have resulted in improved management of 
MTC patients (2). Unfortunately, in our first case the MTC was diagnosed postoperatively after total thyroidectomy performed for multinodular goiter. Preoperative basal serum calcitonin levels and cervical lymph node imaging had not been performed, neither lymph node dissection at first surgery. The second patient was adequately treated surgically; imaging as well as surgery techniques have been improved during the last decades. The fact that this was a low secretory tumor made the monitoring of the second patient challenging.

The first case reported herein depicts a female patient with metastatic MTC in the mediastinum, liver, and thoracic spine who developed breast metastasis 23 years after the initial diagnosis.

Breast is an uncommon site for metastatic carcinoma (with a prevalence of $2 \%$ ) as it consists of fibrous tissue with poor blood supply. The most common extramammary malignancies which metastasize to the breast are lymphomas and hematologic malignancies followed by melanomas, gastric and lung carcinomas, carcinoid tumors, ovarian tumors, liver, kidney, prostate carcinomas, and sarcomas (3). Of all breast metastases only $4 \%$ originate from the thyroid. MTC breast metastasis is exceedingly rare with only 40 cases reported in the literature $(4,5)$. The vast majority concerns women (only one male case reported); the mean age at diagnosis is 43.5 years $(4,5)$. According to the literature, the time interval between the initial diagnosis and the breast metastasis varies from a few days or months to 28 years (mean 7.1 years) $(4,5)$. Differential diagnosis between primary breast carcinoma and breast metastasis of other origin can present difficulties; it is, however, mandatory for planning the appropriate therapeutic approach as well as for the prognosis.

There are no reliable pathognomonic clinical or radiological criteria to distinguish between primary and secondary breast tumors (6). Ultrasonography may reveal a hypoechoic, well-circumscribed mass without acoustic shadow. In mammography, breast metastatic lesions may appear as well-circumscribed or partly irregular masses with increased density and absence of spiculations, microcalcifications, or other signs characteristic for primary carcinomas $(3,6)$. In our case, the nodule of 1 $\mathrm{cm}$ was well visualized on mammography; the absence of microcalcifications or spiculations raised the suspicion of a secondary tumor. Immunohistochemical confirmation with a panel of immunohistochemical markers, including calcitonin and TTF-1 is mandatory as in our case (6).

In our patient, breast metastasis was a late manifestation of advanced MTC disease and is in accordance with relevant reports (7). Furthermore, it appeared 23 years after thyroidectomy. Only one similar case with such late manifestation has been reported (8). Concerning possible mechanisms for the development of breast MTC metastasis, it has been reported that it could be either secondary to hematogenous spread (7) or retrograde lymphatic diffusion from supraclavicular nodes to subclavicular axillary ones and then to the breast possibly after blockage of the lymphatic pathway caused by radiation therapy or lymph node dissection (9). Hematogenous spread hypothesis is more likely as in most cases, similar to our patient, no axillary lymph node involvement is reported.

Although breast metastases usually indicate disseminated disease and poor prognosis, MTC patients with breast metastases tend to have longer survival rates compared to patients having breast metastases originating from other solid tumors and usually undergo excision of metastasis for local disease control (7). In this regard our patient underwent metastasectomy and in conjunction with TKI treatment she is free of disease progression 7 years later.

Concerning the second patient, to our knowledge, this is the first case reported in the literature presenting with both pancreatic and mandible metastases. Only three MTC case reports of pancreatic metastatic involvement have been described $(10,11,12)$ while two cases with MTC metastases in the oral cavity have been reported; one in the anterior maxilla and one in the gingival region $(13,14)$.

Metastatic carcinoma to the pancreas is uncommon representing approximately $4.5 \%$ of all pancreatic masses. Bronchogenic and renal cell carcinoma metastases are the most frequent. Less common sites include, breast, colon, melanoma, liver, and hematopoietic malignancies (12). MTC metastasizes to the pancreas extremely rarely. Our patient did not develop any relevant clinical symptoms and the first pancreatic lesion was identified in a prestaging abdomen MRI and was confirmed with EUS guided FNA biopsy; this procedure is mandatory for a definitedistinction between primary and secondary pancreatic tumors (12). When possible, surgical excision is the preferred treatment for these metastases. However, in our patient pancreatic metastases were multiple and unresectable; so, salvage surgery was ruled out and treatment with vandetanib was initiated. Strangely, the patient developed a mandible metastasis although she had no lung or liver metastases. This coexistence is the first reported in literature.

Metastatic tumors in the oral cavity are generally rare, $1-2 \%$ of all oral malignancies, mandible being the most common (81\% of oral metastases) mainly from breast carcinoma followed by adrenal, colo-rectum, genitalia and thyroid for females (15). Physical examination reveals a 
tender mass which is usually a lytic lesion with irregular margins in imaging, like in our case. Hematogenous spreading is the most likely mechanism involved in the metastasis to the jaw bones. Oral metastases are related with worse prognosis, the mean survival being 7.3 months (15). This was also the case in this MTC associated with inappropriately low-calcitonin levels and accompanied by metastases in both the pancreas and mandible, both features related to poor prognosis.

In conclusion, the cases presented here are two of the very few described in the literature. Although apparently rare, these cases highlight the need for watchful care and prompt diagnosis of uncommon metastatic sites for MTC. Unusual sites of metastatic disease may be present many years after the initial diagnosis, as in our cases. Wherever possible, local therapies (surgery, external beam irradiation) remain the first choice of treatment. TKIs, despite their side effects, are a promising and effective therapeutic tool in patients with advanced MTC; both our patients had disease stabilization under vandetanib treatment for at least 20 months.

\section{Declaration of interest}

No conflict of interest that could be perceived as prejudicing the impartiality of the research reported. Katerina Saltiki is a Senior Editor of Endocrinology Diabetes \& Metabolism Case Reports. Katerina Saltiki was not involved in the review or editorial process for this paper, on which she is listed as an author.

\section{Funding}

This study did not receive any specific grant from any funding agency in the public, commercial or not-for-profit sector.

\section{Patient consent}

Written informed consent for publication of clinical details and/or clinical images was obtained from the patients.

\section{Author contribution statement}

Paraskevi Kazakou wrote the article. George Simeakis, Maria Alevizaki, and Katerina Saltiki revised the article. All authors approved the final content of article. All authors took care of the patients.

\section{References}

1 Wells Jr SA, Asa SL, Dralle H, Elisei R, Evans DB, Gagel RF, Lee N, Machens A, Moley JF, Pacini F, et al. Revised American Thyroid
Association guidelines for the management of medullary thyroid carcinoma. Thyroid 201525 567-610. (https://doi.org/10.1089/ thy.2014.0335)

2 Alevizaki M, Saltiki K, Rentziou G, Papathoma A, Sarika L, Vasileiou V \& Anastasiou E. Medullary thyroid carcinoma: the influence of policy changing in clinical characteristics and disease progression. European Journal of Endocrinology 2012167 799-808. (https://doi.org/10.1530/ EJE-12-0388)

3 DeLair DF, Corben AD, Catalano JP, Vallejo CE, Brogi E \& Tan LK. Non-mammary metastases to the breast and axilla: a study of 85 cases. Modern Pathology 201326 343-349. (https://doi.org/10.1038/ modpathol.2012.191)

4 Mandanas S, Margaritidou E, Christoforidou V, Karoglou E, Geranou C, Chrisoulidou A, Boudina M, Georgopoulos K \& PazaitouPanayiotou K. Breast metastasis from medullary thyroid carcinoma in a male patient: case report and review of the literature. Rare Tumors 20157 5765. (https://doi.org/10.4081/rt.2015.5765)

5 Kanteti AP, Atiya S, Hein A, Cox JL \& Martinez Duarte E. Medullary thyroid carcinoma presenting as metastatic disease to the breast. Case Reports in Pathology 20202020 6138409. (https://doi. org/10.1155/2020/6138409)

6 Nofech-Mozes S, Mackenzie R, Kahn HJ, Ehrlich L \& Raphael SJ. Breast metastasis by medullary thyroid carcinoma detected by FDG positron emission tomography. Annals of Diagnostic Pathology 200812 67-71. (https://doi.org/10.1016/j.anndiagpath.2006.08.004)

7 Ricciato MP, Lombardi CP, Raffaelli M, De Rosa A \& Corsello SM. Metastatic breast involvement from medullary thyroid carcinoma: a clue to consider the need of early diagnosis and adequate surgical strategy. Thyroid 201020 831-832. (https://doi.org/10.1089/ thy.2009.0191)

8 Martínez-Rodríguez I, Banzo I \& Carril JM. Metabolic response demonstrated by 18F-FDG-PET/CT in metastatic medullary thyroid carcinoma under sorafenib therapy. Endocrine 201344 264-265. (https://doi.org/10.1007/s12020-013-9946-6)

9 Marcy PY, Thariat J, Peyrottes I \& Dassonville O. Bilateral breast involvement in medullary thyroid carcinoma. Thyroid 200919 197-199. (https://doi.org/10.1089/thy.2008.0383)

10 Cano JM, Galán R \& López R. Recurrent metastatic medullary thyroid carcinoma: a case of sustained response to prolonged treatment with somatostatin analogues. Thyroid 201727 1450-1455. (https://doi. org/10.1089/thy.2016.0540)

11 Kapur S, Xiao H, Zakowski MF, Hameed MR \& Levin MB. Metastatic medullary thyroid carcinoma and cabozantinib: case series and review of literature. World Journal of Oncology 20145 81-89. (https://doi. org/10.14740/wjon $779 w)$

12 Layfield LJ, Hirschowitz SL \& Adler DG. Metastatic disease to the pancreas documented by endoscopic ultrasound guided fine-needle aspiration: a seven-year experience. Diagnostic Cytopathology 201240 228-233. (https://doi.org/10.1002/dc.21564)

13 Piattelli A, Fioroni M \& Rubini C. Gingival metastasis from a medullary thyroid carcinoma: case report. Journal of Periodontology 200071 112-116. (https://doi.org/10.1902/jop.2000.71.1.112)

14 Shabestari SB, Shirinbak I \& Agha-Hosseini F. Maxillary metastasis of a medullary thyroid carcinoma in a 21-year-old woman 7 years after thyroidectomy. Journal of Oral and Maxillofacial Surgery 201270 1495-1499. (https://doi.org/10.1016/j.joms.2011.04.005)

15 Hirshberg A, Leibovich P \& Buchner A. Metastatic tumors to the jawbones: analysis of 390 cases. Journal of Oral Pathology and Medicine 199423 337-341. (https://doi.org/10.1111/j.1600-0714.1994. tb00072.x) 